\title{
Review of: "Myosin phosphatase target subunit 1 governs integrity of the embryonic gut epithelium to circumvent atresia development in medaka, Oryzias latipes"
}

\author{
Brian Perrino ${ }^{1}$ \\ 1 University of Nevada, Reno
}

Potential competing interests: The author(s) declared that no potential competing interests exist.

This manuscript is focused on the genetic and molecular basis of intestinal atresia (IA); a birth defect of the intestine resulting in complete intestinal blockage during embryonic development. An idiopathic lack of local blood supply to the developing embryonic gut because of a vascular defect is usually responsible for most cases of IA. However, familial cases and a higher incidence among 21 trisomy patients indicate genetic factors may also play a role. Thus, the molecular mechanism underlying IA appears to be etiologically heterogeneous and remains largely unknown.

Here, the embryonic development of the Japanese rice fish medaka is used as a model of intestinal development. They identified a medaka mutant in which IA develops during embryogenesis, isolated through $\mathrm{N}$-ethyl-N-nitrosourea (ENU) mutagenesis screening. Positional cloning identified a nonsense lossof function (LOF) mutation in MYPT1. During embryogenesis, the endoderm cells behaved properly in the MYPT1 LOF mutant to form the gut precursor. However, the gut precursor in the MYPT1 LOF mutant became discontinued along the anteroposterior axis of the intermediate portion resulting in IA, just after the formation of a gut lumen was completed. These findings indicate that MYPT1 function in intestinal epithelial cells is necessary for gut development in the medaka model.

MYPT1 is a regulatory subunit of myosin light chain phosphatase (MLCP), regulating the specificity and activity of the PP1 catalytic subunit toward MRLC. Phosphorylation of MYPT1 by ROCK inhibits MLCP activity. In addition, CPI-17 phosphorylated by PKC is able to bind with high affinity to the active site of PP1 bound to MYPT1 in MLCP, further inhibiting MLCP activity towards MRLC. Thus, consistent with a loss of MYPT1 function, intestinal epithelial cells of the mutant at the site of the lesion displayed a hypercontracted phenotype, due to increased MRLC pS19 phosphorylation, and an increased F-actin/Gactin ratio.

The mutation in MYPT1 due to ENU resulted in the loss of the LZ motifs at the C-terminus. These LZ motifs are responsible for binding CGMP protein kinase (PRKG) to MYPT1, resulting in MYPT1 phosphorylation, and activation of MLCP, dephosphorylation of MRLC pS19, and relaxation. Thus, MLCP activity in this ENU MYPT1 mutant would be expected to be extremely low, resulting in loss of function.

To show that loss of MYPT1 function results in IA, an N-terminal MYPT1 truncation LOF mutant was 
generated. Experiments using this MYPT1 mutant showed that consistent with a loss of MYPT1 function, intestinal epithelial cells of the mutant at the site of the lesion displayed increased MRLC pS19 phosphorylation, an increased F-actin/G-actin ratio, and a hypercontracted phenotype. These findings indicate that loss of MYPT1 function in intestinal epithelial cells results in IA in the medaka model. However, no experiments examining MRLC S19 phosphorylation in the MYPT1 C-terminal LZ motif truncation mutant were presented. The authors acknowledge that the mechanism responsible for such a specific loss of MYPT1 function is unknown, but probably involves specific changing patterns of gene expression during embryonic development. In addition, whether a loss of MYPT1 function in gut epithelial cells occurs from the lack of local blood supply to the developing embryonic gut that causes IA is unknown, but these findings do shed light on a possible genetic factor for IA.

Since the goal of this report was to show that loss of MYPT1 function in epithelial cells of the developing gut of medaka embryos results in IA, the authors accomplished this goal. However, the loss of MYPT1 function by the $\mathrm{N}$-terminal truncation and the C-terminal truncation mutants are due to completely different mechanisms, and raise interesting and important questions about the regulation of gut epithelium morphogenesis by epithelial cell MYPT1 and MLCP.

The N-terminal MYPT1 LOF mutant is due to the absence of full length MYPT1 expression. In this situation, the phosphatase catalytic subunit of MLCP is not localized to myosin by MYPT1, nor is the specificity of the active site for MRLC pS9 arranged by MYPT1. Interestingly, in gut smooth muscle cells, loss of MYPT1 did not result in any obvious phenotypic changes, as MBS 85 was able to bind to the phosphatase catalytic subunit and substitute for MYPT1.

The C-terminal truncation mutant has only lost the LZ PRKG-binding motif, so presumably this MYPT1 fragment is still bound to the phosphatase catalytic subunit in MLCP, and still contains its regulatory phosphorylation sites. In this situation, MLCP is subject to inhibition by MYPT1 phosphorylation at Thr696 and Thr853. Interestingly, however, this MYPT1 mutant would not be subject to activation by PRKG phosphorylation of Ser695; which inhibits the inhibitory Thr696 phosphorylation. Therefore, one might expect this MYPT1 mutant to be hyperphosphorylated at its inhibitory Thr696 site and MLCP activity to be severely inhibited. It would be very interesting to determine the role of NO, CGMP, and PRKG in regulating MYPT1 phosphorylation and MLCP activity in wild-type medaka gut epithelial cells during embryonic development. Overall, this is an important paper for revealing possible genetic factors that contribute to IA during embryonic development, and important for indicating the possible role of NO-cGMP signaling in regulating MYPT1 phosphorylation and MLCP activity in the embryonic development of gut epithelial cells. 